\title{
Fractional Fourier Based Waveform for a Joint Radar-Communication System
}

\author{
Domenico Gaglione*, Carmine Clemente*, Christos V. Ilioudis*, \\ Adriano Rosario Persico*, Ian K. Proudler ${ }^{\dagger}$, and John J. Soraghan* \\ ${ }^{*}$ University of Strathclyde, CeSIP, EEE, 204, George Street, G1 1XW, Glasgow, UK \\ E-mail: domenico.gaglione, carmine.clemente, c.ilioudis, adriano.persico, j.soraghan@ strath.ac.uk \\ $\dagger$ School of Electronic, Electrical and Systems Engineering, Loughborough University, Leicestershire, UK \\ E-mail: i.k.proudler@lboro.ac.uk
}

\begin{abstract}
The increasing demand of spectrum resources and the need to keep the size, weight and power consumption of modern radar as low as possible, has led to the development of solutions like joint radar-communication systems. In this paper a novel Fractional Fourier Transform (FrFT) based multiplexing scheme is presented as joint radar-communication technique. The FrFT is used to embed data into chirp sub-carriers with different time-frequency rates. Some optimisation procedures are also proposed, with the objective of improving the bandwidth occupancy and the bit rate and/or Bit Error Ratio (BER). The generated waveform is demonstrated to have a good rejection to distortions introduced by the channel, leading to low BER, while keeping good radar characteristics compared to a widely used Linear Frequency Modulated (LFM) pulse with same duration and bandwidth.
\end{abstract}

\section{INTRODUCTION}

Spectrum congestion is increasingly becoming a significant issue, due to the ever greater demand on bandwidth for different kind of RF applications [1]. Radar and broadband communications are two of the most onerous applications, since they both take advantage of wide bandwidth to achieve high data rate and enhanced resolution, respectively.

In some scenarios radars need to share data with other systems, i.e. in a surveillance system where early warning messages must be sent to a central node or in a Multiple-Input Multiple-Output (MIMO) radar in which different nodes need to communicate with each other. An efficient and effective solution to the bandwidth sharing is the use of a joint radarcommunication technique, whose main idea is to use the same transceiver for radar and communication purposes. This also meets the low-SWaP (Size, Weight and Power consumption) requirements, since no dedicated antenna and transceiver are needed for the communication task in addition to the radar sensor.

Possible implementations of a joint radar-communication system include the exploitation of waveform diversity [2], signal separation through techniques such as Successive Interference Cancellation (SIC) [3] and spectrum sharing [4], [5]. More complex solutions consist of embedding the data to be transmitted into the radar waveform [6]. These can be categorized in Time Division Duplex (TDD) mode techniques [7], which generally use Frequency Modulated Continuous Wave (FMCW) radar waveforms, and Frequency Division Duplex (FDD) mode techniques. Example of FDD mode approach with single-carrier waveform is [8], in which a Direct Sequence Spread Spectrum (DSSS) technique is proposed as method to avoid mutual interference between radar and communication data. In [9] the authors proposed a Linear Frequency Modulated (LFM) Minimum Shift Keying (MSK) waveform design while in [10] and [11] an approach based on the orthogonality between up-chirp and down-chirp signals able to reach $1 \mathrm{Mb} / \mathrm{s}$ at a maximum range of $2 \mathrm{Km}$ is presented and validated, respectively. Orthogonal Frequency Division Multiplexing (OFDM) waveforms [12] are widely used in radar systems nowadays for their high time-bandwidth product, and they also represent a natural candidate for the implementation of a multi-carrier joint radar-communication system [13]. An example is the joint radar-communication system presented in [14] and developed for automotive application, which is able to reach a very high bit rate of $20 \mathrm{Mb} / \mathrm{s}$. In [15], [16] the authors also demonstrated the capability of such a multiplexing technique for covert and multi-user communication.

In this paper a novel Fractional Fourier Transform (FrFT) [17], [18] based waveform design is presented for joint radarcommunication systems (Co-Radar). The proposed multiplexing scheme can generally be employed whatever there is the need to perform radar tasks and send information to one or multiple receivers, especially in application where nodes are far away from eachc other. FrFT waveforms have already been demonstrated to be suitable for MIMO radar systems [19][22], as well as for wireless RF communication [23]-[26], underwater communication [27], [28], interference excision [29] and encryption [30].

The remainder of the paper has the following structure. Section II presents the novel multiplexing technique, while some optimisation procedures aimed at maximising the band occupancy and improve the performance in terms of bit rate and/or bit error ratio are presented in Section III. Radar and communication performance are evaluated in Section IV, while Section V concludes the paper.

\section{FRFT BASED CO-RADAR}

The proposed FrFT based Co-Radar waveform design is a multiplexing scheme. Like the OFDM uses the Fast Fourier Transform (FFT) to map the in-phase and quadrature (IQ) samples coming from a digital modulator (i.e. M-PSK) to different frequency sub-carriers, the FrFT based multiplexer maps the IQ symbols to different chirp, or LFM, sub-carriers with different time-frequency rates. Before explaining the proposed waveform design in detail, the FrFT is briefly presented. 

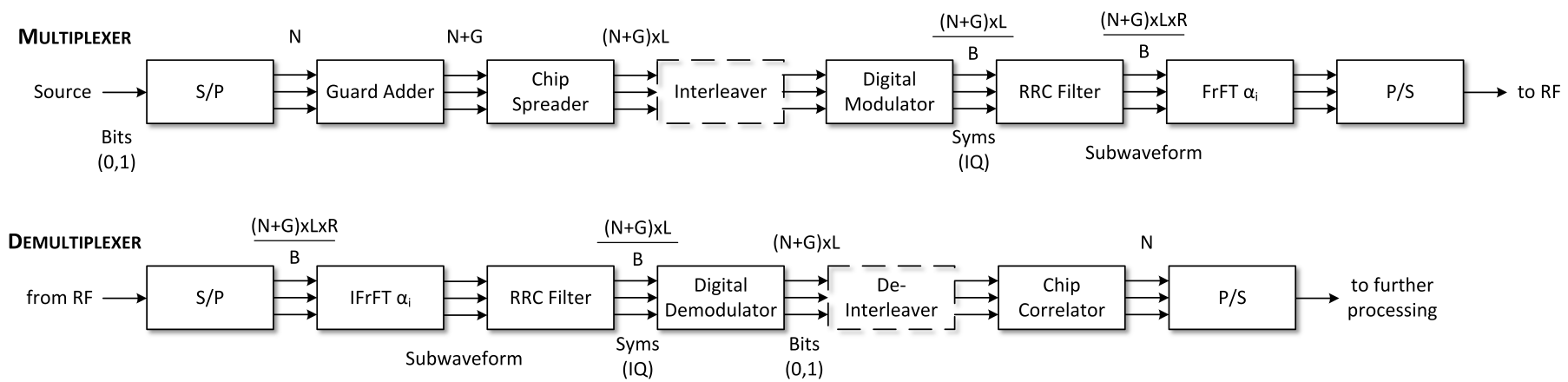

Figure 1. Block diagram of the (top) Multiplexer and (bottom) Demultiplexer of the proposed Co-Radar waveform design framework.

\section{A. Fractional Fourier Transform}

The Fractional Fourier Transform (FrFT) belongs to the class of linear Time-Frequency Representations (TFRs), and it was firstly introduced in [17]. It is a generalisation of the ordinary Fourier transform and can be considered as a rotation by an arbitrary angle, $\phi$, in the time-frequency plane. Letting $x(u)$ be an arbitrary signal of length $U$, its $\alpha^{t h}$-order discrete FrFT is defined as [18]:

$$
X_{\alpha}[u]=\sum_{u^{\prime}=-U / 2}^{U / 2} K_{\alpha}\left[u, u^{\prime}\right] x\left[u^{\prime}\right]
$$

where

$$
\alpha=\frac{2}{\pi} \times \phi
$$

is the fractional order and $K_{\alpha}\left[u, u^{\prime}\right]$ is the FrFT kernel, defined as [18]:

$$
K_{\alpha}\left[u, u^{\prime}\right]= \begin{cases}A_{0} e^{j \pi\left(u^{2}+u^{\prime 2}\right) \cot \phi} \times & \text { if } \phi \neq m \pi \\ e^{-j \pi 2 u u^{\prime} \csc \phi} & \text { if } \phi=2 m \pi \\ \delta\left[u-u^{\prime}\right] & \text { if } \phi=2 m \pi+\pi \\ \delta\left[u+u^{\prime}\right] & \end{cases}
$$

where $A_{0}=\frac{e^{j \frac{\phi}{2}}}{\sqrt{j \sin \phi}}, \delta(\cdot)$ is the Dirac delta function, $j=$ $\sqrt{-1}$ and $m \in \mathbb{Z}$ is an integer. The FrFT is an invertible linear transform continuous in the angle $\phi$, which satisfies the basic conditions for it to be meaningful in the time-frequency plane.

\section{B. Multiplexer}

The multiplexer of the proposed Co-Radar waveform design framework is shown at the top of Figure 1. The serialto-parallel $(\mathrm{S} / \mathrm{P})$ block is used to divide the long sequence of bits coming from the source into $C$ segments of $N$ bits each, where $C$ is the number of chirp sub-carriers to use. At each segment, $G$ guard bits are added at the end of the sequence in order to compensate the group delay introduced by the Root Raised Cosine (RRC) filter. Then the sequence is spread by using a chip sequence; the chosen chip sequence is a $L$-long Barker code, which leads to a spread sequence of $(N+G) \times L$ bits.

An interleaver may be used to mitigate the Inter-Carrier Interference (ICI); this solution is discussed in more detail later in the paper.

The digital modulator maps a series of $B$ bits in one of the $M=2^{B}$ possible complex symbols belonging to the chosen modulation scheme (i.e. QAM, PSK), leading to a $(N+G) \times L / B$ long symbol sequence. The modulation scheme and the cardinality of its alphabet $M$ can be adaptively chosen according to the conditions of the channel.

The RRC filter is used to minimise the Inter-Symbol Interference (ISI) that may be caused by the channel. For efficiency, it is implemented as a multirate filter that upsamples the output by a factor $R$, leading to a final sequence of $(N+G) \times L \times R / B$ samples.

The $C$ subwaveforms obtained after the RRC filter are then mapped to different chirp sub-carriers uniformly spaced in the time-frequency domain. Since the FrFT is periodic in $\alpha$ with period 4 , the uniformly spaced sub-carriers are obtained by choosing the $i$-th fractional order equal to $\alpha_{i}=i \bar{\alpha}, i=$ $0, \ldots, C-1$, where $\bar{\alpha}=\frac{2}{C}$. Finally, the parallel-to-serial (P/S) block combines the chirp modulated subwaveforms by adding them together.

Examples of axes normalised spectrograms of FrFT based Co-Radar waveforms are shown in Figure 2, with (a) $C=10$ and (b) $C=20$ chirp sub-carriers, respectively. $B W$ and $\tau$ are the bandwidth and the duration of the pulse.

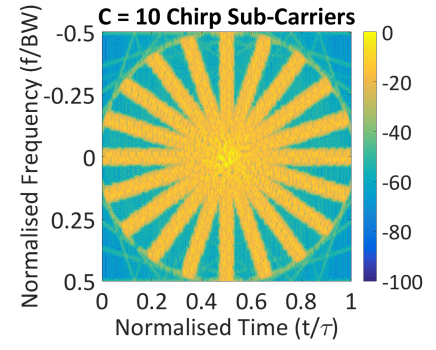

(a)

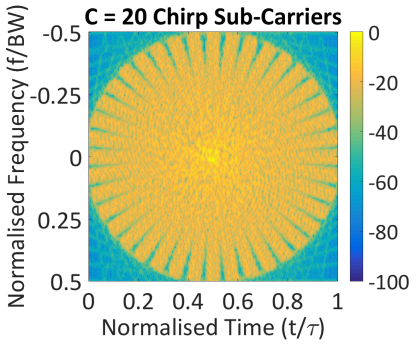

(b)
Figure 2. Spectrograms of FrFT based Co-Radar waveforms, with (a) $C=10$ and (b) $C=20$ chirp sub-carriers.

\section{Demultiplexer}

The demultiplexer is shown at the bottom of Figure 1. The S/P block splits and redirects the acquired signal, whose length is $(N+G) \times L \times R / B$ samples, to $C$ different IFrFT blocks that perform the inverse FrFT transform. Each sequence is then input of the RRC filter, which also down-samples the subwaveform by a factor $R$. The digital demodulator translates the $(N+G) \times L / B$ long sequence of symbols in a sequence of $(N+G) \times L$ bits, according to the modulation employed.

At this point, a de-interleaver may be placed which performs the inverse operation of the interleaver. 
The chip correlator block correlates the input spread sequence with the $L$-long Barker chip code used in transmission to extract the $N$ bits of information, exploiting both the low correlation side lobes and the knowledge that the peaks of the correlation occur every $L$ samples. Finally, the P/S block reconstructs the original stream by combining the $N$-long bit sequences coming from the $C$ different parallel branches.

\section{WAVEFORM OPTIMISATION}

In this section two waveform optimisation procedures are described: the adaptive duration of the subwaveforms aims to efficiently occupy the available bandwidth, whereas the introduction of either a guard time or an interleaver has the objective of minimising the Inter-Carrier Interference (ICI).

\section{A. Subwaveform Adaptive Duration}

As shown in Figure 2, the FrFT based Co-Radar pulse does not occupy the entire available bandwidth since it is clearly enclosed in a circle of radius one half in normalised units. In order to maximise the bandwidth occupancy, it is possible to consider subwaveforms with different time durations on varying the fractional order. This also leads to an increase of the bit rate with no effect on the bit error ratio, at a cost of a slightly higher design complexity.

An example of a longer subwaveform rotated of an angle $\phi_{i}=\pi / 4$ and whose bandwidth is $B W_{s u b}$ is shown in Figure 3(a). The fraction of additional time with respect to the duration of the pulse, $\tau_{\text {plus }}$, is:

$$
\tau_{\text {plus }_{i}}=\frac{\tau_{i}-\tau}{2 \tau}
$$

where $\tau_{i}$ is the duration of the $i$-th subwaveform given by:

$$
\tau_{i}=\left\{\begin{array}{cc}
\frac{\tau}{\left|\cos \left(\phi_{i}\right)\right|} & \phi_{i} \in\left[0, \frac{\pi}{4}\left[\cup \left[\frac{3 \pi}{4}, \pi[\right.\right.\right. \\
\frac{\tau}{\left|\sin \left(\phi_{i}\right)\right|} & \phi_{i} \in\left[\frac{\pi}{4}, \frac{3 \pi}{4}[\right.
\end{array}\right.
$$

$i=0, \ldots, C-1$. Thus, the number of bits that the longer $i$-th subwaveform can accommodate is given by:

$$
(N+G)_{i}=\left\lfloor\frac{\tau_{i} B W}{L R} B\right\rfloor \text { s.t. } \frac{(N+G)_{i} \times L}{B} \in \mathbb{N}
$$

where the function $\lfloor\cdot\rfloor$ gives the largest integer not greater than the argument and $\mathbb{N}$ is the set of natural numbers.

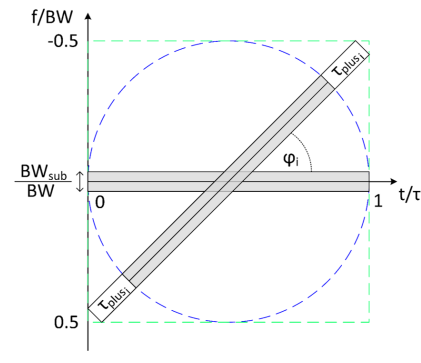

(a)

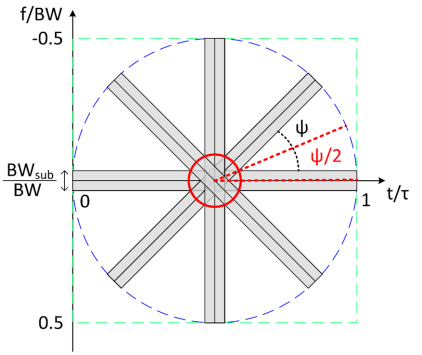

(b)
Figure 3. Waveform optimisation: representation of (a) subwaveforms with different duration on varying the fractional order and (b) the ICI and the guard time $T_{G}$.

Equation (2) shows a linear relationship between the fractional order $\alpha$ and the rotation angle $\phi$. When subwaveforms with different durations are considered, this equation becomes nonlinear and can be written as:

$$
\alpha_{i}=\frac{2}{\pi} \times\left[\phi_{i}+\gamma\left(\phi_{i}, \tau_{p_{l u s}}\right)\right]
$$

where

$$
\begin{aligned}
& \gamma\left(\phi_{i}, \tau_{\text {plus }_{i}}\right)= \\
& \quad \cos ^{-1}\left[\cos \phi_{i}\left(\frac{\eta}{1+2 \tau_{\text {plus }_{i}}}+\frac{2 \tau_{\text {plus }_{i}}}{\eta\left(1+2 \tau_{\text {plus }_{i}}\right)}\right)\right]
\end{aligned}
$$

with

$$
\eta=\sqrt{1+\left(1+2 \tau_{\text {plus }}\right)^{2} \tan ^{2} \phi_{i}}
$$

An example of a spectrogram of a FrFT based Co-Radar waveform with adaptive duration is shown in Figure 4. The red circle indicates the time-frequency region occupied by the pulse when no optimisation is applied. In terms of bit rate, the adaptive duration optimisation leads to an improvement of about $10 \%$.

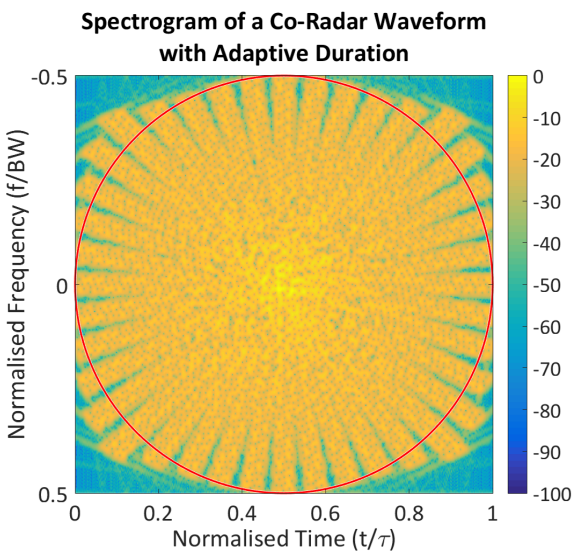

Figure 4. Spectrogram of a FrFT Based Co-Radar Waveform with Adaptive Duration and $C=20$ chirp sub-carriers.

\section{B. ICI Mitigation}

Due to the nature of chirps, when more than one sub-carrier is used there is an overlapping area with centre at the zero frequency and half duration of the pulse that produces ICI. In this section two ICI mitigation approaches are proposed for the developed waveform design.

Guard Time In order to avoid data loss, the time-frequency region affected by ICI is not used for the transmission of bits of information; it rather carries guard bits. This can be achieved by slightly modifying the guard adder block in Figure 1, such that it adds both $G_{2}$ bits at the middle of the sequence to mitigate the ICI and $G_{1}$ bits at the end in order to compensate the group delay introduced by the RRC filter, leading to an overall guard band width of $G=G_{1}+G_{2}$. Moreover, a guard remover block after the chip correlator at the end of the demultiplexer is included.

The duration in time of the guard, $T_{G}$, is graphically represented by the diameter of the red circle in Figure 3(b). The value of $T_{G}$ depends on the inter-carrier separation angle $\psi=\phi_{i+1}-\phi_{i}, i=0, \ldots, C-2$ and the bandwidth of the subwaveform $B W_{s u b}$, and it is approximately equal to:

$$
T_{G} \approx \frac{B W_{s u b}}{B W} \csc \left(\frac{\psi}{2}\right) \tau
$$


The guard time $T_{G}$ translates in a guard band width of $G_{2}$ bits given by:

$$
G_{2}=\left\lceil\frac{N+G_{1}}{1-\frac{T_{G}}{\tau}}\left(\frac{T_{G}}{\tau}\right)\right\rceil
$$

where the function $\lceil\cdot\rceil$ gives the smallest integer not less than the argument.

Interleaver Another approach to mitigate the ICI regards the use of an interleaver. As shown in Figure 3(b), the interference is localised around the centre of rotation of the waveform. This means that it generates a burst of errors, affecting a small group of bits that can be dealt with the use of a suitable interleaving technique.

An interleaver/de-interleaver pair placed before the digital modulator in transmission and after the digital demodulator in reception (Figure 1), spreads the burst errors across the entire sequence. A basic interleaver can be used, implemented as a matrix which is filled by rows and emptied by columns. By setting the number of rows to $N+G$, and the number of columns to the length of the Barker code, $L$, the errors are equally distributed across the $N+G$ bits of the original sequence.

\section{PERformance ANALysis}

In this section the radar performance and the communication performance of the Co-Radar system are investigated. The former is evaluated in terms of range resolution, Doppler resolution and Side Lobe Levels (SLLs), and compared with a pulse radar system which occupies the same bandwidth with a LFM pulse. The communication performance is examined in terms of Bit Error Ratio (BER) for different channel conditions and different configurations of the system, assuming to send uncoded data (i.e. without any forward error correction).

Moreveor, the radar is supposed to be working in a monostatic configuration with a Pulse Repetition Frequency (PRF) of $3 \mathrm{kHz}$, which leads to an unambiguous range of $50 \mathrm{Km}$, sufficient for applications such as surveillance.

\section{A. Radar Performance}

In order to assess the radar performance of the FrFT CoRadar waveform, its Ambiguity Function (AF) is examined. Moreover, the zero-Doppler and zero-Delay cuts obtained from the proposed multiplexing scheme are presented and compared with those of a typical LFM waveform with same duration and bandwidth.

Since the transmitted radar signal depends on the data to be sent, it varies throughout the different transmissions. To examine its $\mathrm{AF}$ characteristics an average of them is considered through 100 Monte Carlo runs. The available bandwidth is set to $B W=560 \mathrm{MHz}$ and $\tau=10 \mu \mathrm{s}$ is the width of the pulse.

The zero-Delay and zero-Doppler cuts of the LFM and CoRadar AFs, with both $C=10$ and $C=20$ sub-carriers, are shown in Figure 5. Regarding the zero-Delay cut, the LFM has a narrower main lobe compared to the Co-Radar waveform but suffers from higher Side Lobe Levels (SLLs). It can be therefore inferred that the Co-Radar waveforms provide worse Doppler resolution but lower SLL interference compared to the LFM sequence. No differences in terms of resolution and first SLL are appreciable when comparing the Co-Radar waveforms with different number of sub-carriers; however, when moving

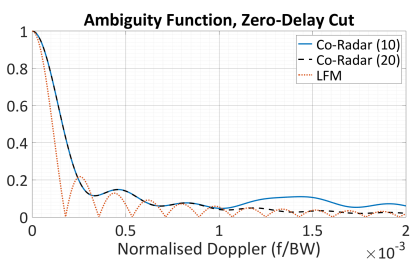

(a)

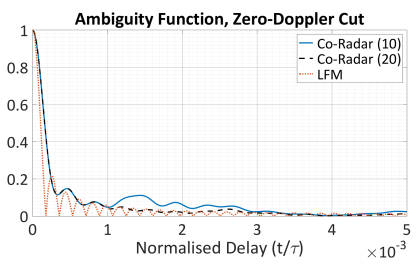

(b)
Figure 5. (a) Zero-Delay and (b) Zero-Doppler cuts of the Co-Radar and LFM waveforms' AF.

from $C=10$ to $C=20$ the AF's floor generally decreases, as shown also in Figure 6. Same observations can be made for the zero-Doppler cut: the LFM waveform has superior range resolution but higher SLLs compared to the Co-Radar ones. This can be explained by the non-constant envelope of the Co-radar waveform, which leads to broader main lobes but lower SLLs in both cuts.

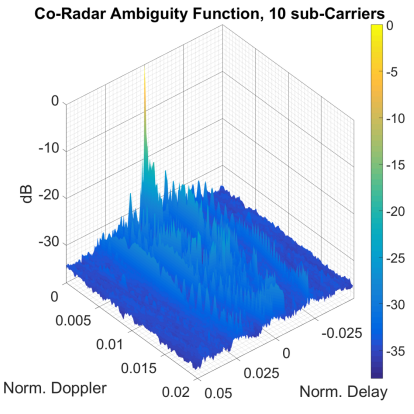

(a)

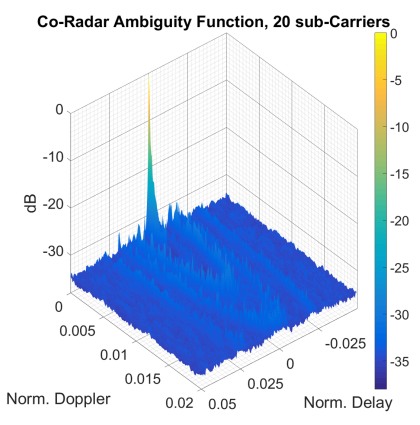

(b)
Figure 6. Co-Radar waveform AF's with (a) $C=10$ and (b) $C=20$ sub-carriers.

Table I summarises the radar performance parameters for both Co-Radar and LFM waveforms. Doppler and range resolution are taken by assuming a $-3 d \mathrm{~B}$ reference level.

The proposed approach also has an actual impact on the pulse-to-pulse clutter attenuation, thanks to the low crosscorrelation between consecutive pulses.

TABle I. Radar Performance Parameters

\begin{tabular}{|l|c|c|c|}
\hline & Co-Radar (10) & Co-Radar (20) & LFM \\
\hline Doppler Res. & $64.27 \mathrm{kHz}$ & $64.29 \mathrm{kHz}$ & $44.33 \mathrm{kHz}$ \\
\hline Range Res. & $34.50 \mathrm{~cm}$ & $34.53 \mathrm{~cm}$ & $24.09 \mathrm{~cm}$ \\
\hline Zero-Delay SSL & $-16.59 \mathrm{~dB}$ & $-16.61 \mathrm{~dB}$ & $-13.26 \mathrm{~dB}$ \\
\hline Zero-Doppler SSL & $-16.62 \mathrm{~dB}$ & $-16.53 \mathrm{~dB}$ & $-13.26 \mathrm{~dB}$ \\
\hline
\end{tabular}

\section{B. Communication Performance}

For the analysis of the communication performance, the signal is assumed to experience a slow-flat fading, therefore a time-invariant narrowband channel model is considered. Let $\mathbf{s}_{\mathrm{tx}}$ be the vector which contains the transmitted signal samples. The received signal can be written as:

$$
\mathbf{s}_{\mathbf{r x}}=\mathbf{h} \circ \mathbf{s}_{\mathbf{t x}}+\boldsymbol{\zeta}
$$

where $\mathbf{h}$ is the vector that contains the channel coefficients, $\boldsymbol{\zeta}$ is the white Gaussian noise and the operator $\circ$ indicates the Hadamard, or entrywise, product.

The complex elements of the vector $\mathbf{h}$ belongs to a statistical distribution depending on the propagation path. In addition 
to the Additive White Gaussian Noise (AWGN) scenario for which $h=1$, three other cases are considered. When no Line of Sight (LOS) path exists, the channel coefficients $\mathbf{h}$ are drawn from a Rayleigh distribution with scale parameter $\sqrt{2} / 2$. In the case of existence of LOS path, the channel is modelled as Rician with a Rice factor of 5 . Finally, in order to take into account shadowing and diffraction that can occur in bad weather conditions, a combination of Rice and Lognormal is considered [31]. In this case the channel coefficients are obtained as the product between a Rice process normalised in power and a Lognormal variable, whose associated Gaussian variable has a standard deviation of 4 (this is often referred to as the " $d \mathrm{~B}$ spread").

Assuming that the received signal has been equalised, the communication performance is evaluated in terms of BER vs SNR for the four different types of channel, for the two above mentioned ICI mitigation techniques and for 10 and 20 sub-carriers. The pulse width $\tau$ is set to $10 \mu \mathrm{s}$, the system bandwidth, $B W$, is $560 \mathrm{MHz}$ while $N+G=64$. The length of the chip sequence, $L$, is 7 and the up-sampling factor, $R$, is 25 . An $M$-PSK modulation is used with $M=4$; the bandwidth of each subwaveform depends on this choice and the configuration of the RRC filter. It can be evaluated as $B W_{\text {sub }}=R_{s}(\beta+1)$, where $R_{s}=\frac{(N+G) L}{B \tau}$ is the symbol rate and $\beta=0.4$ is the RRC roll-off coefficient.

A Monte Carlo approach has been used to calculate the mean value of the BER over 1000 tests. For the ICI mitigation approach which uses a guard time, the latter has been chosen equal to:

$$
\bar{T}_{G}=\rho T_{G}
$$

where $T_{G}$ is the minimum guard time derived in the previous section and $\rho$ is a constant which lies in $\left[1, \tau / T_{G}\right]$, sometimes expressed in percentage.

Figure 7 shows the BER obtained for $C=20$ and assuming that (a) $\rho=1.1$ and (b) $\rho=1.2$. The performance generally improves as the SNR increases and independently of the type of channel. Slightly better performance is obtained when a larger guard time is used. However, the bit rate is reduced in this case, at $2.358 \mathrm{Mb} / \mathrm{s}$, while it increases up to $2.514 \mathrm{Mb} / \mathrm{s}$ when $\rho=1.1$.

The BER obtained for $C=10$ is shown in Figure 8. Again, it decreases as the SNR increases and better performance is obtained when the guard time is larger. The bit rates in these two cases are $1.638 \mathrm{Mb} / \mathrm{s}$ and $1.596 \mathrm{Mb} / \mathrm{s}$ for $\rho=1.1$ and $\rho=1.2$, respectively.

Moreover, comparing Figure 7 and Figure 8 it is clear how, for the same values of SNR, the BER becomes smaller when the number of chirp sub-carriers changes from 20 to 10 , result of the lesser ICI.

Figure 9 shows the performance in terms of BER when the interleaver is used as technique for ICI mitigation. The figure shows the performance when the number of carriers is (a) $C=10$ and (b) $C=20$. As expected, the BER decreases as the SNR increases and better performance is achieved for 10 sub-carriers with respect to 20 , despite the fact that the bit rate drops from $3.660 \mathrm{Mb} / \mathrm{s}$ with $C=20$ to $1.830 \mathrm{Mb} / \mathrm{s}$ with $C=$ 10. More interesting is the comparison between the two ICI mitigation approaches. For all the considered types of channel, when 10 sub-carriers are used the interleaver always offers better performance, together with a higher bit rate: $1.830 \mathrm{Mb} / \mathrm{s}$ rather than $1.638 \mathrm{Mb} / \mathrm{s}$ and $1.596 \mathrm{Mb} / \mathrm{s}$. The two techniques

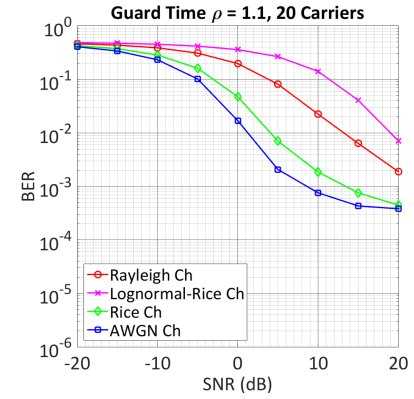

(a)

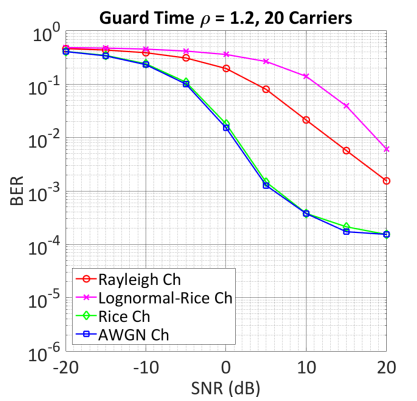

(b)
Figure 7. BER obtained with $C=20$ sub-carriers with adaptive duration of the subwaveforms and guard time greater of (a) $10 \%$ and (b) $20 \%$ than the minimum value.

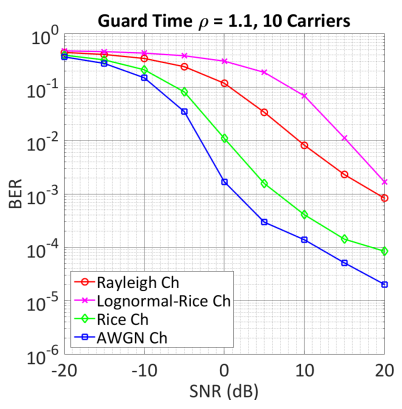

(a)

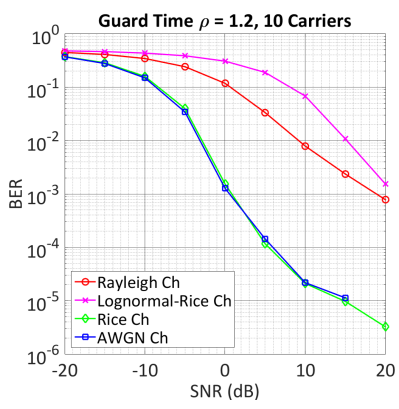

(b)
Figure 8. BER obtained with $C=10$ sub-carriers with adaptive duration of the subwaveforms and guard time greater of (a) $10 \%$ and (b) $20 \%$ than the minimum value.

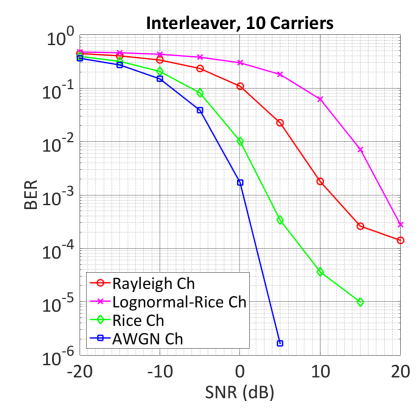

(a)

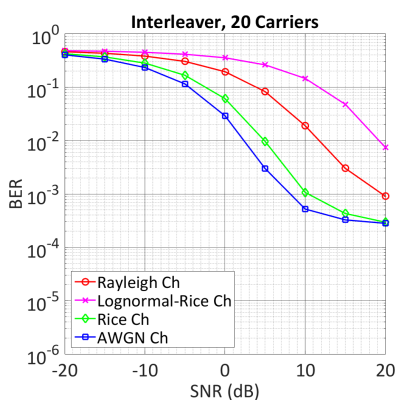

(b)
Figure 9. BER obtained with (a) $C=10$ and (b) $C=20$ sub-carriers with adaptive duration of the subwaveforms, when the interleaver is used as method for ICI mitigation.

give very similar results in terms of BER when 20 sub-carriers are used, however the interleaver allows us to reach a bit rate $45 \%$ higher than the bit rate achievable with the guard time technique and $\rho=1.1$.

Moreover, assuming that the radar meets the low-SWaP paradigm requirements with a transmitted average power of $50 W$ (peak power $32 d \mathrm{~B}$ with a duty cycle of $3 \%$ ), it is safe to assume that the SNR at the communication receiver ranges between $10 d \mathrm{~B}$ and $40 \mathrm{~dB}$. Hence, even in the worst case, which arises when the communication receiver appears in the side lobe of the antenna's radar, the BER is lower than $10^{-2}$ when an interleaver is used and for AWGN, Rayleigh and Rice channels.

\section{Conclusion}

A novel Fractional Fourier Transform based waveform design for joint radar-communication systems has been presented. Similar to the OFDM, the proposed multiplexing 
scheme embeds data to be sent into chirp sub-carriers with different time-frequency rates. The developed framework is fully scalable, since it is easily adaptable to the available bandwidth, the length of the pulse, the conditions of the channel. Optimisation procedures have also been described aiming to improve the bandwidth occupancy and the bit rate/bit error rate.

Simulation analyses have validated the feasibility of the novel multiplexing scheme and demonstrated the good radar characteristics of the generated waveform compared to a LFM pulse with same duration and bandwidth. On the communication side, a bit rate up to $3.660 \mathrm{Mb} / \mathrm{s}$ has been achieved, which is an improvement over the state-of-the-art for medium range joint radar-communication systems. However, the maximum data rate strictly depends on the radar requirements.

\section{ACKNOWLEDGMENT}

This work was supported by the Engineering and Physical Sciences Research Council (EPSRC) Grant number EP/K014307/1 and the MOD University Defence Research Collaboration in Signal Processing.

\section{REFERENCES}

[1] H. Griffiths, "The Challenge of Spectrum Engineering," in 2014 11th European Radar Conference (EuRAD), Oct 2014, pp. 1-4.

[2] A. Chiriyath, B. Paul, G. Jacyna, and D. Bliss, "Inner Bounds on Performance of Radar and Communications Co-existence," IEEE Transactions on Signal Processing, vol. PP, no. 99, pp. 1-1, 2015.

[3] A. Hassanien, M. Amin, Y. Zhang, and F. Ahmad, "A Dual Function Radar-Communications System Using Sidelobe Control and Waveform Diversity," in 2015 IEEE Radar Conference (RadarCon), May 2015, pp. $1260-1263$.

[4] A. Turlapaty and Y. Jin, "A Joint Design of Transmit Waveforms for Radar and Communications Systems in Coexistence," in 2014 IEEE Radar Conference, May 2014, pp. 0315-0319.

[5] R. Romero and K. Shepherd, "Friendly Spectrally Shaped Radar Waveform With Legacy Communication Systems for Shared Access and Spectrum Management," IEEE Access, vol. 3, pp. 1541-1554, 2015.

[6] L. Han and K. Wu, "Joint Wireless Communication and Radar Sensing Systems - State of the Art and Future Prospects," IET Microwaves, Antennas Propagation, vol. 7, no. 11, pp. 876-885, August 2013.

[7] L. Han and K. Wu, "Radar and Radio Data Fusion Platform for Future Intelligent Transportation System," in 2010 European Radar Conference (EuRAD), Sept 2010, pp. 65-68.

[8] S. Xu, Y. Chen, and P. Zhang, "Integrated Radar and Communication Based on DS-UWB," in The Third International Conference on Ultrawideband and Ultrashort Impulse Signals, Sept 2006, pp. 142-144.

[9] X. Chen, X. Wang, S. Xu, and J. Zhang, "A Novel Radar Waveform Compatible with Communication," in 2011 International Conference on Computational Problem-Solving (ICCP), Oct 2011, pp. 177-181.

[10] M. Roberton and E. Brown, "Integrated Radar and Communications Based on Chirped Spread-Spectrum Techniques," in 2003 IEEE MTTS International Microwave Symposium Digest, vol. 1, June 2003, pp. 611-614.

[11] G. N. Saddik, R. Singh, and E. Brown, "Ultra-Wideband Multifunctional Communications/Radar System," IEEE Transactions on Microwave Theory and Techniques, vol. 55, no. 7, pp. 1431-1437, July 2007.

[12] N. Levanon, "Multifrequency Radar Signals," in 2000 IEEE International Radar Conference, 2000, pp. 683-688.

[13] J. Zhao, K. Huo, and X. Li, "A Chaos-Based Phase-Coded OFDM Signal for Joint Radar-Communication Systems," in 2014 12th International Conference on Signal Processing (ICSP), Oct 2014, pp. 19972002
[14] C. Sturm and W. Wiesbeck, "Waveform Design and Signal Processing Aspects for Fusion of Wireless Communications and Radar Sensing," Proceedings of the IEEE, vol. 99, no. 7, pp. 1236-1259, July 2011.

[15] S. Surender and R. Narayanan, "UWB Noise-OFDM Netted Radar: Physical Layer Design and Analysis," IEEE Transactions on Aerospace and Electronic Systems, vol. 47, no. 2, pp. 1380-1400, April 2011.

[16] Y. Sit, C. Sturm, and T. Zwick, "One-Stage Selective Interference Cancellation for the OFDM Joint Radar-Communication System," in 2012 The 7th German Microwave Conference (GeMiC), March 2012, pp. 1-4.

[17] V. Namias, "The Fractional Order Fourier Transform and its Application to Quantum Mechanics," IMA Journal of Applied Mathematics, vol. 25, no. 3, pp. 241-265, 1980 .

[18] H. M. Ozaktas, Z. Zalevsky, and M. A. Kutay, The Fractional Fourier Transform with Applications in Optics and Signal Processing. Wiley, Chichester, 2001.

[19] C. Clemente, I. Shorokhov, I. Proudler, and J. Soraghan, "Radar Waveform Libraries Using Fractional Fourier Transform," in 2014 IEEE Radar Conference, May 2014, pp. 0855-0858.

[20] C. Clemente, C. Ilioudis, D. Gaglione, K. Thompson, S. Weiss, I. Proudler, and J. Soraghan, "Reuse of Fractional Waveform Libraries for MIMO Radar and Electronic Countermeasures," in 2014 6th International Symposium on Communications, Control and Signal Processing (ISCCSP), May 2014, pp. 505-508.

[21] C. Ilioudis, C. Clemente, I. Proudler, and J. Soraghan, "Constant Envelope Fractional Fourier Transform Based Waveform Libraries for MIMO Radar," in 2014 Sensor Signal Processing for Defence (SSPD), Sept 2014, pp. 1-5.

[22] C. Ilioudis, C. Clemente, I. Proudler, and J. Soraghan, "Performance Analysis of Fractional Waveform Libraries in MIMO Radar Scenario," in 2015 IEEE Radar Conference (RadarCon), May 2015, pp. 11191124.

[23] M. Martone, "A Multicarrier System Based on the Fractional Fourier Transform for Time-Frequency-Selective Channels," IEEE Transactions on Communications, vol. 49, no. 6, pp. 1011-1020, Jun 2001.

[24] C. Wang, Z. Zhang, S. Li, and Q. Fu, "Transform Domain Communication System Based on Random-Ambiguity and Fractional Fourier Transform," in 2006 6th International Conference on ITS Telecommunications Proceedings, June 2006, pp. 194-197.

[25] X.-J. Sha, R. hui Wen, and X. Qiu, "A New Multiple-Access Method Based on Fractional Fourier Transform," in 2009 Canadian Conference on Electrical and Computer Engineering (CCECE), May 2009, pp. 856859.

[26] Y. Chi, S. Zhang, and J. Shi, "Fractional Fourier Domain Communication System: System Structure and Signal Modeling," in 2011 6th International ICST Conference onCommunications and Networking in China (CHINACOM), Aug 2011, pp. 560-564.

[27] Y. Chen, P. Cai, and Y. Wang, "A New Underwater Acoustic Communication System Based on Fractional Fourier Transform," in 2010 IEEE International Conference on Information and Automation (ICIA), June 2010, pp. 413-418.

[28] Y. Chen, C. Clemente, J. J. Soraghan, and S. Weiss, "Partial Fraction Fourier Transform (PFRFT)-OFDM for Underwater Acoustic Communication," in 2015 23rd European Signal Processing Conference (EUSIPCO), Sept 2015.

[29] O. Akay and G. Boudreaux-Bartels, "Broadband Interference Excision in Spread Spectrum Communication Systems via Fractional Fourier Transform," in 1998 Thirty-Second Asilomar Conference on Signals, SystemsComputers, vol. 1, Nov 1998, pp. 832-837 vol.1.

[30] X. Qiu, X.-J. Sha, Q. hua Shen, and X. li Wu, "Fractional Fourier Transform Based Secure Communication System," in 2010 6th International Conference on Wireless Communications Networking and Mobile Computing (WiCOM), Sept 2010, pp. 1-4.

[31] G. Corazza, C. Ferrarelli, and F. Vatalaro, "A Rice-Lognormal Terrestrial and Satellite Channel Model," in 1994 Third Annual International Conference on Universal Personal Communications, Sep 1994, pp. 155159. 